\title{
La mejora de la competencia lingüística desde la interacción comunicativa: una propuesta para la enseñanza bilingüe
}

\author{
Víctor CANTERO GARCÍA \\ Universidad Pablo de Olavide \\ cantero91@hotmail.com
}

Recibido: octubre 2012

Aceptado: junio 2014

\section{RESUMEN}

En el presente artículo se pretende dar respuesta a una de las carencias más claras en el actual modelo de la enseñanza bilingüe practicado en los IES, a saber: la necesidad de mejorar los niveles de competencia lingüística de los alumnos en L2 por medio del refuerzo de las actividades de interacción comunicativa en dicha lengua.

Tras la identificación de esta laguna en la práctica docente diaria, el autor busca en su entorno aquellas prácticas educativas que en la actualidad tratan de paliarla. Tomando como referencia esas buenas prácticas, se concluye la colaboración ofreciendo a los docentes de las Secciones Bilingües una serie de propuestas didácticas de fácil aplicación en sus clases de la ESO, las cuales tienen como propósito incrementar los niveles de interacción comunicativa de sus alumnos mediante un uso funcional y práctico de la L2.

Palabras clave: mejora, competencia lingüística, interacción comunicativa, enseñanza bilingüe.

The improvement of linguistic competence from communicative interaction: a proposal for bilingual education

\begin{abstract}
This article seeks to answer on of the most clear shortcomings in the current model of bilingual education as practiced in the IES, namely: the need to improve the skill levels of students in linguistic competence in the L2 by means of strengthening communicative interaction activities in that language.

After indentifying this gap in the daily teaching practice, the author seeks in its environment those educational practices that currently seek to adress. Drawing on these good practices, it is concluded collaboration offering bilingual teachers Sections a series of educational proposals of easy application in their classes in the ESO, which are intended to increase the levels of their student's communicative interaction through a funtional and practical use of the L2.
\end{abstract}

Keywords: improvement, linguistic competence, interaction of communication, bilingual education. 


\section{L'enrichissement de la compétence linguistique par l'interaction communicative: une proposition pour l'enseignement bilingue}

\section{RÉSUMÉ}

Dans cet article, nous allons faire remarquer l'un des manques du modèle actuel de l'enseignement bilingue pratiqué dans nos lycées : le besoin d'améliorer les niveaux de compétence des élèves en L2 à travers le renforcement des activités d'interaction communicative dans la langue en question. Après avoir identifié ce manque dans la pratique quotidienne, l'auteur cherchera dans son environnement professionnel ces pratiques éducatives qui, actuellement, cherchent à finir avec ce manque en compétence communicative. On prendra comme référence ces bonnes pratiques. Ce papier se termine en offrant aux enseignants des sections bilingues une série de propositions didactiques de facile application lors de leurs cours de l' E.S.O qui auront comme but d'améliorer les niveaux d'interaction communicative de leurs élèves par l'usage fonctionnel et pratique de la L2.

Most-clés: l'amélioration, maîtrise de la langue et l'interaction communicative, l'enseignement bilingue.

SUMARIO: 1 Introducción 2 De la detección de las carencias a la búsqueda de soluciones 3. Buscando la inspiración en propuestas exitosas posibles de emular 4. Elaboración de propuestas propias válidas para mejorar la competencia lingüística de los alumnos/as en L2. 5 Conclusiones. 6. Bibliografía.

\section{INTRODUCCIÓN}

Han pasado algunos cursos desde que la Consejería de Educación de la Junta de Andalucía aprobase el Plan de Fomento del Prurilingüismo en Andalucía ${ }^{1}$, aludimos a un Proyecto Educativo que desde esa fecha ha pretendido introducir en los IES andaluces un determinado modelo de enseñanza bilingüe. Yo participé en la primera convocatoria para la selección de Centros Bilingües ${ }^{2}$ y mi IES fue seleccionado, a consecuencia de lo cual ejercí como Coordinador de su Sección Bilingüe (español-inglés) e intenté poner en práctica la Programación Didáctica elaborada como un elemento más del Plan de Centro. Han pasado más de cinco años desde esa fecha y sigo vinculado a tareas que tienen mucho que ver con la enseñanza bilingüe: Coordinador de un Proyecto Comenius 1.1 (Intercambios Escolares), Profesor de L1 en Sección Bilingüe, con perfil y cualificación profesional en L2, entre otras. Por ello, creo que ha llegado el momento de hacer un alto en el camino y reflexionar sobre los aciertos y carencias de una propuesta

${ }^{1}$ Según Acuerdo de 22 de marzo de 2005, del Consejo de Gobierno, (BOJA, no 65, de 5.4.2005).

${ }^{2}$ La realizada por Orden de la CEJA de 6.4.2005, para el curso 2005/2006, (BOJA, nº 66, de 6.4.2005) 
educativa con la que se pretende enseñar a los alumnos de la ESO una serie de contenidos de las Áreas no Lingüísticas (ANLs) en inglés.

Sin duda, los logros son muchos y variados, y de ellos ya hemos dado buena cuenta en las preceptivas Memorias Finales de cada curso, así como en las evaluaciones internas y externas que se han realizado en relación con nuestro Proyecto de Innovación Educativa. Sin embargo, no todo son luces, pues una de las sombras más reiteradas es el lento progreso de nuestros alumnos/as en la mejora de sus capacidades de interacción comunicativa en L2, tanto en las relaciones profesor/alumno como en los intercambios verbales practicados por los propios estudiantes. Realizadas las correspondientes mediciones de este factor, los niveles de mejora no se incrementan de forma significativa año tras año, lo cual no deja de ser preocupante. Con el fin de ilustrar lo dicho, adjunto como muestra alguno de los cuadros de resultados correspondientes a los dos últimos cursos de la experiencia.

\section{Cuadro-resumen I}

(Medición de niveles de incremento en \% de la mejora de interacción comunicativa en L2. Curso 2012/2013)

\begin{tabular}{|c|c|c|}
\hline & $\begin{array}{c}\text { Interacción } \\
\text { profesor/alumno }\end{array}$ & $\begin{array}{c}\text { Interacción } \\
\text { alumno/alumno }\end{array}$ \\
\hline $\mathbf{1}^{\mathbf{0}}$ ESO-Bilingüe & $26,3 \%$ & $22,5 \%$ \\
\hline $\mathbf{1}^{\mathbf{0}}$ ESO no Bilingüe & $23,5 \%$ & $20,5 \%$ \\
\hline $\mathbf{2}^{\mathbf{0}}$ ESO Bilingüe & $21,3 \%$ & $22,4 \%$ \\
\hline $\mathbf{2}^{\mathbf{0}}$ ESO no Bilingüe & $21,6 \%$ & $19,7 \%$ \\
\hline $\mathbf{3}^{\mathbf{0}}$ ESO Bilingüe & $22,7 \%$ & $20,3 \%$ \\
\hline $\mathbf{3}^{\mathbf{0}}$ ESO no Bilingüe & $21,2 \%$ & $22,8 \%$ \\
\hline $\mathbf{4}^{\mathbf{0}}$ ESO Bilingüe & $26,5 \%$ & $20,1 \%$ \\
\hline $\mathbf{4}^{\mathbf{0}}$ ESO no Bilingüe & $22,7 \%$ & $19,6 \%$ \\
\hline
\end{tabular}

Cuadro-resumen II

(Medición de niveles de incremento en \% de la mejora en interacción comunicativa en L2. Curso 2013/2014)

\begin{tabular}{|c|c|c|}
\hline & $\begin{array}{c}\text { Interacción profesor- } \\
\text { alumno }\end{array}$ & $\begin{array}{c}\text { Interacción alumno- } \\
\text { alumno. }\end{array}$ \\
\hline $\mathbf{1}^{\mathbf{0}}$ ESO Bilingüe & $32,5 \%$ & $33,7,4 \%$ \\
\hline $\mathbf{1}^{\mathbf{0}}$ ESO no Bilingüe & $30,6 \%$ & $31,6 \%$ \\
\hline $\mathbf{2}^{\mathbf{0}}$ ESO Bilingüe & $29,5 \%$ & $28,6 \%$ \\
\hline
\end{tabular}




\begin{tabular}{|c|c|c|}
\hline $\mathbf{2}^{\mathbf{0}}$ ESO no Bilingüe & $30,1 \%$ & $29,7 \%$ \\
\hline $\mathbf{3}^{\mathbf{0}}$ ESO Bilingüe & $29,5 \%$ & $29,7 \%$ \\
\hline $\mathbf{3}^{\mathbf{0}}$ ESO no Bilingüe & $31,7 \%$ & $30,6 \%$ \\
\hline $\mathbf{4}^{\mathbf{0}}$ ESO Bilingüe & $33,5 \%$ & $34,3 \%$ \\
\hline $\mathbf{4}^{\mathbf{0}}$ ESO no Bilingüe & $32,1 \%$ & $32,6 \%$ \\
\hline
\end{tabular}

De la información numérica se desprende que en este intervalo se ha avanzado muy poco en el dominio de la interacción comunicativa por parte de los alumnos sometidos a pruebas y controles, así como que la diferencia en relación con dicho dominio de la L2 por parte de los alumnos bilingües y monolingües es muy escasa. La crudeza de los datos me hizo pensar en tomar medidas para abordar este evidente desajuste entre el tiempo y el esfuerzo dedicado en las aulas a las prácticas de interacción comunicativa en L2 y los resultados obtenidos por los alumnos que estudian ANLs en inglés, tanto en las pruebas de "speaking" como en los "listening". Todos los miembros de la Sección Bilingüe éramos conscientes de que si la finalidad de la enseñanza bilingüe impartida a nuestros alumnos/as se centraba en capacitarlos para que contasen con una mayor fluidez verbal, expresiva e intercomunicativa en la Lengua Extranjera (LE) algo había que hacer para remediar esta carencia. Y el fruto del tiempo dedicado a reflexionar sobre este asunto, a cómo subsanar esta laguna conforma el contenido del presente artículo, cuyo objetivo es ofrecer al docente que imparte ANLs en inglés en Secciones Bilingües algunas alternativas válidas para aumentar esos bajos niveles de interacción comunicativa detectados en los estudiantes.

\section{DE LA DETECCIÓN DE LAS CARENCIAS A LA BÚSQUEDA DE SOLUCIONES}

Detectado el fallo todo era cuestión de ponerse manos a la obra y motivación para ello no nos faltaba. ¿Pero en qué dirección debíamos caminar? ¿Cómo podíamos echar a andar sin errar el periplo? Y la mejor forma de evitar los titubeos, a mi juicio, consistía en cerciorarnos de que la mejora de la interacción comunicativa en L2 es uno de los pilares de cualquier modalidad de enseñanza bilingüe. En un segundo lugar, y despejada esta duda, podríamos abordar la búsqueda de otros ensayos, de otras propuestas didácticas que ya han enfocado sus esfuerzos con éxito en esta misma dirección. Y como colofón, resultará imprescindible que aportemos nuestras propias aportaciones, al objeto de ayudar al profesorado a superar este bache.

Empecemos, por tanto, nuestro itinerario volviendo los ojos a los fundamentos del modelo de enseñanza bilingüe — metodología CLIL— practicado en los IES. Ha 
llovido mucho desde que la Conferencia de Luxemburgo, 1923, rechazase el bilingüismo temprano y recomendase la lengua familiar como idioma vehicular de contenidos escolares. Una posición que fue confirmada en 1952, cuando la UNESCO citó en París a un grupo de expertos, los cuales se ratificaron en la idea de desaconsejar la introducción de una segunda lengua a edades muy tempranas. Hoy estas posiciones están superadas, entre otras cosas, porque tal como señala Ignasi Villar: "se apoyaban en un modelo psicolingüístico que presuponía que las lenguas del alumno bilingüe se almacenaban en compartimentos distintos en la mente del hablante" (2010, p. 1) por lo que no existía relación alguna entre L1 y L2. Y a la superación de estos presupuestos contribuyeron, sin duda, los trabajos de Penfield y Lambert y su grupo de estudio de la Universidad McGill en Montreal (Canadá), allá por los años 60 del siglo pasado. Tras múltiples pruebas demuestran que los alumnos bilingües, incluso los tempranos, experimentan mayores progresos académicos en el medio escolar que los monolingües. Y ello era debido a que los sujetos bilingües poseen una competencia lingüística en cada una de las lenguas que dominan, de lo que se desprende que ambas lenguas interactúan en la mente del hablante, el cual suma a las habilidades y destrezas - bilingüismo aditivo- que posee en la lengua materna las que va adquiriendo en la L2 en el medio escolar. Por ello la incorporación de la L2 en los programas de inmersión lingüística no se ve ya como una amenaza para la consolidación del aprendizaje de la L1, al contrario, todo lo que el niño/a aprende y asimila en la L2 en cuanto a lectura, escritura y comunicación verbal se traslada a la L1, lo que refuerza el aprendizaje de ambas. Pero no volvemos la vista hacia los trabajos de Lambert por mera erudición, antes al contrario lo hacemos para percatarnos de que, en la misma raíz de los programas de inmersión lingüística ideados por él y que triunfan en los años 70 , se encuentra la constatación de que los alumnos mejoran sensiblemente su competencia comunicativa en la L2 sin que se perjudique el aprendizaje y el dominio de la L1.

Sin embargo, tiene que llegar el modelo psicolingüístico de J. Cummins (1997) para que se produzca un verdadero avance en el modelo de enseñanza bilingüe. Cummins sostiene que no existen almacenes mentales separados para cada una de las lenguas del sujeto bilingüe, sino que lo que existe es una sola competencia lingüística general que se puede vehicular hacia una $u$ otra lengua de forma indistinta. Y esta competencia general se relaciona básicamente con el dominio del instrumento lingüístico, el cual es el medio más privilegiado para propiciar los intercambios sociales. Y aquí es donde queríamos llegar, a saber: independiente de la forma que adopte cada lengua, el lenguaje es un instrumento básico de comunicación, por ello existen unas reglas de uso de cada lenguaje que se relacionan con la competencia lingüística de cada hablante. Lo que dicho de otro modo supone que en la adquisición de un nuevo lenguaje predomina la negociación de los contenidos de interacción adulto-niño, de lo que se desprende que la situación comunicativa es un factor esencial en los programas de inmersión, precursores de los actuales modelos de enseñanza bilingüe. De este marco 
negociador se desprenden una serie de beneficios para el hablante bilingüe, siendo el primero de ellos el incremento de su conocimiento lingüístico de la L2 al tener que negociar el sentido y el significado de sus producciones habladas. La segunda ventaja consiste en que el hablante bilingüe es capaz de entender la intención comunicativa de los mensajes que recibe en L2 porque usa su propia competencia lingüística para codificarlos y descodificarlos. Y el tercer elemento favorable se centra en la actitud positiva que los alumnos que participan en la enseñanza bilingüe muestran hacia el aprendizaje de la L2. Una actitud que es determinante a la hora de explicar sus progresos en el dominio escrito y hablado tanto de la L1 como de la L2.

Por lo tanto, de esta primera aproximación a los fundamentos de la enseñanza bilingüe podemos deducir que, tal como señala Milagros Pérez Calvo: "en la enseñanza de contenidos mediante la LE se contemplan registros de la lengua específicos de la materia que van a permitir una mejora considerable de la competencia comunicativa del alumno que reciba este tipo de enseñanza" (2010, p. 2). Un comentario que subraya nuestra creencia en la importancia que la interacción comunicativa tiene ya desde los mismos cimientos de la enseñanza bilingüe, pues de carecer de dicha destreza comunicativa al sujeto le resulta muy difícil utilizar correctamente ambas lenguas en situaciones concretas de comunicación. Por ello el uso de la LE para el estudio de contenidos en áreas como Matemáticas, Ciencias Sociales o Música contribuye a que los alumnos/as asimilen con mayor rapidez el sistema lingüístico de la L2, toda vez que su aprendizaje se estudia en distintos contextos y a través de diferentes situaciones de comunicación. Llegados a este punto ya estamos en disposición de comprender que la enseñanza bilingüe no tiene como objetivo prioritario solo mejorar los niveles de competencia lingüística de nuestros alumnos, sino que lo que pretende es ampliar la capacidad de sus relaciones sociales gracias al dominio de dichas lenguas. En otras palabras, nos referimos a sus habilidades para interactuar con las demás personas en situaciones y contextos diferentes. De ello se deriva que la metodología CLIL que empleamos en las Secciones Bilingües encuentre su refrendo en afirmaciones como la de Gennesse, el cual manifiesta que: "la efectividad de los programas de integración de lenguas y contenidos depende, sin ningún tipo de duda, de la interacción entre el docente y sus estudiantes" (1987, p. 160). Una interacción que pasa por la negociación de los significados que se ponen en práctica y las correspondientes estrategias de consenso que ayudan a la mutua comprensión de los participantes en el acto comunicativo. Hablamos de una negociación de significados que tiene sus puntos en común con la teoría de la adquisición de las segundas lenguas postulada por Krashen (1985), llamada "input hipótesis" o "teoría del input comprensible". Una propuesta que insiste en la necesidad de que sea el profesor quien fomente la interacción comunicativa entre sus alumnos, evitando en todo momento que el discurso en el aula sea unidireccional.

Finalizamos nuestro recorrido por los principios de la enseñanza bilingüe analizando las aportaciones que la psicodidáctica realiza en pro de nuestra causa, es 
decir, que las situaciones y experiencias comunicativas en varias lenguas refuerzan el componente interrelacional de la enseñanza bilingüe. En este sentido, tal como sostiene Josetxo Madariaga:

La escuela actual tiene dos restos significativos que afrontar en la actualidad, de un lado la creciente heterogeneidad lingüística de nuestra sociedad, y de otro, la compresión de fenómenos comunicativos y de interacción que se producen en las experiencias educativas bilingües. (2010, p. 2)

Son precisamente estos retos los que incitan a la psicodidáctica a interesarse por las experiencias en enseñanza bilingüe, pues estas prácticas educativas han evolucionado mucho de la mano de la psicolingüística. Hoy ya no se ve el fenómeno bilingüe como un hecho circunscrito a las tareas de adquisición o aprendizaje de ambas lenguas, sino que tal como sostienen Siguan y Mackey (1986): "hoy no es posible estudiar el bilingüismo en el individuo humano sin tener en cuenta la función que cumplen las lenguas que habla en la sociedad a la que pertenece, por lo tanto sin tener presente el bilingüismo de la sociedad en la que vive" (1986, p. 234). Por ello resulta evidente que la persona se convierte en bilingüe debido a su necesidad de comunicarse con las personas que no hablan su lengua. De aquí se desprende el hecho de que, para evaluar la eficacia de un programa bilingüe desde la óptica psicodidáctica, no sea suficiente con analizar el nivel de conocimiento que el alumno posee de ambas lenguas, sino que también es preciso medir su capacidad para utilizarlas en la práctica con normalidad. Se trata de medir la correcta utilización de las lenguas en los diversos contextos sociales, una cuestión bastante compleja y que requiere de un mayor esfuerzo investigador. La psicodidáctica afronta esta tarea y una vez superados los postulados de la inmersión lingüística, propios del modelo bilingüe canadiense, nos ofrece el resultado de las investigaciones de Álvarez y Del Río (1990), según las cuales la función del lenguaje no solo se refiere al acto del habla, sino que también puede tener una connotación semiótica, lo que implica que el alumno debe saber usar la L2 en relación con sus diferentes funciones. Por ello ambos autores sostienen que al lenguaje se le debe atribuir gran importancia no solo por su significado o por su capacidad para ayudar a la interacción, sino también por el sentido que tiene el habla en si misma. Una opinión que redobla nuestro afán por encontrar en la filosofía de la enseñanza bilingüe una respuesta a nuestros interrogantes, a saber: que este modelo de enseñanza no solo se basa en la comprensión de la L1 y L2 por parte del hablante, sino que aprender una lengua de un modo realmente efectivo implica el uso diario de la misma con un nivel de éxito, algo que se supone en los estudiantes que emplean ambas lenguas de forma vehicular en los procesos de enseñanza-aprendizaje. Esta suposición implica un idéntico tratamiento de las lenguas que forman parte del currículo escolar de la ESO, hecho que facilita la 
transferencia de competencias lingüísticas entre ellas. En este sentido, tal como nos precisa, J. M. Serra:

El Equipo Directivo de cada Centro Bilingüe debe contar con los criterios orientativos claros y coherentes que regulan la presencia de las lenguas en el currículo, tanto en cuanto al grado de presencia de las mismas, como en relación con el trabajo que los profesores deben realizar en el aula. (1989, p. 60)

La realización de este periplo nos ha convencido de que nuestro propósito de potenciar los valores de la interacción comunicativa en la enseñanza bilingüe cuenta con un refrendo suficiente; no solo por parte de los teóricos e investigadores, sino que también encuentra eco en las prácticas educativas realizadas en este ámbito.

\section{BUSCANDO LA INSPIRACIÓN EN PROPUESTAS EXITOSAS POSIBLES DE EMULAR}

El siguiente paso en nuestro itinerario se centra en localizar experiencias educativas que están vigentes en la actualidad y que se centran en potenciar la mejora de los niveles de comunicación e interacción comunicativa en la ESO. Y mi primera sorpresa al respecto fue descubrir la existencia del Proyecto Atlántida del OAPEE (Organismo Autónomo de Programas Europeos) del Ministerio de Educación. Se trata de un Proyecto Educativo que centra sus trabajos en la elaboración de propuestas prácticas para la mejora de la comunicación lingüística en L1 y L2 para cada una de las siete destrezas básicas establecidas en el MCER (Marco Común Europeo de Referencia de las Lenguas). Desarrollar aquí todos los pormenores del Proyecto Atlántida ${ }^{3}$ excede los propósitos de la presente colaboración. Por ello, del mismo modo tan solo tomamos aquellos aspectos que subrayan el desarrollo de la destreza productiva "hablar", que es la que hace a nuestro caso. Y para que dicha destreza pueda ser desarrollada y puesta en práctica con acierto en las aulas de la ESO, a fin de que mejoren los índices de interacción comunicativa de los alumnos, se deben cumplir, cuando menos, las siguientes once condiciones:

1.- Realizar una enseñanza con eficacia y equidad, que mejore a todos los alumnos/as.

2.- Priorizar el uso funcional de la lengua: apostar por su uso comunicativo y social.

3.- Respetar la disparidad de los niveles de dominio lingüístico en el aula.

4.- Fomentar la colaboración de todas las áreas curriculares en la misma tarea y compartir materiales.

\footnotetext{
${ }^{3}$ Para ello remitimos al lector a la consulta del texto completo del Proyecto en José Moya Otero y Florencio Luengo Orcajo, (2009), Proyecto Atlántida, Madrid, ME-OPAEE.
} 
5.- Priorizar actividades comunicativas con su correspondiente evaluación.

6.- Ligar la mejora de la enseñanza a la satisfacción del desarrollo profesional del docente.

7.- Promover redes de escuelas: intercambios de buenas prácticas.

8.- Integrar y coordinar familia, escuela y comunidad.

9.-Integrar currículum formal, informal y no formal, lo académico y lo extraescolar.

10.- Incorporar el uso de las NNTT como recurso y soporte textual e hipertextual.

11.- Prestar atención a las relaciones entre las competencias básicas, y de la competencia lingüística con la competencia cívico-social.

Si aplicamos estas condiciones al desarrollo de la sexta destreza: destreza productiva "hablar", dicha destreza ha de ser concretada en los siguientes cinco niveles básicos de integración de las competencias básicas en el curriculum:

a) En las tareas reales del aula: es responsabilidad de cada profesor seleccionar aquel tipo de actividades y aquella propuesta metodológica que motive a sus alumnos/as a ejercitarse más y mejor en la interacción comunicativa en el aula.

b) En las relaciones con el resto de los elementos curriculares: corresponde a cada docente establecer dentro de su aula qué relaciones existen entre su esfuerzo por incrementar la competencia en comunicación lingüística de sus estudiantes y el logro que estos consigan en relación al dominio de las competencias básicas. Enlazando estas con los objetivos, los contenidos, los criterios de evaluación y su programación didáctica habitual.

c) En cuanto a la forma de plantear las tareas (metodología): es competencia de cada profesor buscar aquellos métodos que estimulen la participación conversacional de sus alumnos/as en el aula y que incrementen su competencia lingüística.

d) Medir la importancia que tienen los agentes externos en la acción educativa diaria: el docente no puede obviar el hecho de que la familia, los amigos, el medio social y la calle mediatizan sus esfuerzos por estimular la interacción comunicativa de sus alumnos en L1 y L2.

e) La evaluación de las destrezas comunicativas y sociales de los alumnos/as: es tarea primordial del profesor elaborar aquellos instrumentos más operativos para evaluar los grados de mejora en esta parcela de la competencia comunicativa de sus estudiantes.

Establecidos estos cinco niveles básicos de integración, nuestra labor cuenta con una orientación muy clara, toda vez que tal como sostienen los responsables del 
Proyecto Atlántida para la C. A. Andaluza, en el aparado sobre la "práctica de la comunicación lingüística" queda muy claro que:

La competencia en comunicación lingüística es la madre de todas las competencias. Es a través del lenguaje como adquirimos los conocimientos y las competencias básicas. Dominar las destrezas lingüísticas es la condición necesaria para tener el éxito en el resto de las competencias. Y ese dominio de las competencias lingüísticas solo es posible adquirirlo, ejercitándolas en los contextos adecuados. Es imprescindible pues, transformar nuestra práctica docente de forma que nuestros alumnos/as puedan hablar y escribir, interactuar oralmente y por escrito; en definitiva, ejercitar las siete destrezas lingüísticas de una forma regular, controlada y planificada adecuadamente, a lo largo de su vida escolar. (2009, p. 30)

Pues bien, estas recomendaciones del Proyecto Atlántida se concretan en una serie de estrategias lingüísticas para el fomento de la interacción comunicativa en todas las áreas curriculares de la ESO, especialmente en las lingüísticas. Dichas estrategias para la L1 y la L2 quedan recogidas en los siguientes cuadros:

\section{Cuadro-resumen I}

(Esquema de estrategias de enseñanza aprendizaje y contenidos a desarrollar para potenciar la destreza productiva: "hablar" en la $\mathrm{L1}$ en la $\mathrm{ESO}^{4}$

\begin{tabular}{|c|c|c|c|}
\hline $\begin{array}{l}\text { ¿Qué desarrollar? } \\
\text { (Subcompetencias) }\end{array}$ & $\begin{array}{l}\text { ¿Cómo desarrollar? } \\
\text { (Estrategias para la } \\
\text { enseñanza/aprendizaje) }\end{array}$ & $\begin{array}{l}\text { ¿Con quién } \\
\text { contar? } \\
\text { (Apoyo familia, } \\
\text { comunidad, etc) }\end{array}$ & $\begin{array}{l}\text { Contexto: } \\
\text { (Área } \\
\text { trabajo, } \\
\text { Dpto., Ciclo, etc) } \\
\end{array}$ \\
\hline $\begin{array}{l}\text { * Que el alumno explique sus } \\
\text { vivencias, ideas, } \\
\text { conocimientos, incorporando } \\
\text { en su discurso características } \\
\text { culturales, de experiencia vida } \\
\text { en diferentes ámbitos: entre } \\
\text { iguales, familia, escolares, etc. } \\
\text { * Que exprese su opinión sobre }\end{array}$ & $\begin{array}{l}\text { * Dinamizando sesiones en las que } \\
\text { cada estudiante informe cómo ha } \\
\text { organizado y categorizado la } \\
\text { información que ha registrado } \\
\text { durante el desarrollo de una } \\
\text { determinada exposición oral. }\end{array}$ & 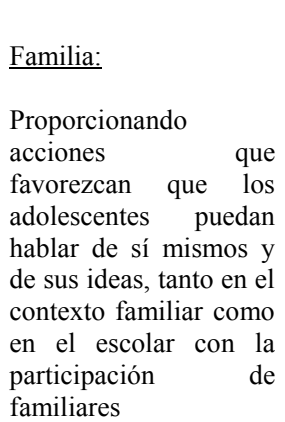 & $\begin{array}{l}\text { * A realizar por el } \\
\text { Equipo Docente que } \\
\text { imparte docencia en } \\
\text { L1 y L2. }\end{array}$ \\
\hline
\end{tabular}

${ }^{4}$ Fuente: Revista Escuela, Monográfico no 3/ diciembre, 2009. Competencias Básicas en la práctica. Comunicación Lingüística. Herramientas para el trabajo del profesorado, pp. 5 y 7. 


\begin{tabular}{|c|c|c|}
\hline $\begin{array}{l}\text { los temas de debate, tras } \\
\text { consultar, entre otros } \\
\text { materiales, sus notas tomadas } \\
\text { durante el desarrollo de una } \\
\text { exposición oral. } \\
\text { * Que pueda distanciarse del } \\
\text { texto, distinguiendo lo que "se } \\
\text { dice" de lo que se interpreta } \\
\text { que "quiere decir", } \\
\text { *Exponer oralmente un relato } \\
\text { (película, cuento, novela, etc.) } \\
\text { guiado por distintas intenciones } \\
\text { comunicativas. } \\
\text { * Expresar oralmente sus ideas } \\
\text { sobre el contenido de ciertos } \\
\text { textos. } \\
\text { * Disponer de estrategias } \\
\text { expositivas para presentar el } \\
\text { contenido de un texto en } \\
\text { distintos contextos } \\
\text { comunicativos. } \\
\text { * Expresar opiniones acerca de } \\
\text { determinados hechos, noticias, } \\
\text { textos. } \\
\text { * Utilizar la lengua cooficial, si } \\
\text { la hubiere, como vehículo de } \\
\text { cohesión la escuela y su } \\
\text { entorno social. }\end{array}$ & $\begin{array}{l}\text { * Acompañando el pensamiento de } \\
\text { los estudiantes, mediante preguntas } \\
\text { estratégicas, para que ellos y ellas } \\
\text { expliciten el análisis que hacen de } \\
\text { determinados actos de habla (lo que } \\
\text { "se dice" y lo que se "quiere } \\
\text { decir"). }\end{array}$ & $\begin{array}{l}\text { (exposiciones de sus } \\
\text { trabajos, debates, } \\
\text { informes } \\
\text { investigaciones, etc). } \\
\\
\text { Comunidad: } \\
\text { Propiciando que los/las } \\
\text { adolescentes tengan un } \\
\text { papel activo en la } \\
\text { organización de } \\
\text { distintas actividades en } \\
\text { el contexto de la } \\
\text { comunidad con la } \\
\text { intención de que les } \\
\text { sirvan de plataforma } \\
\text { para que ellos y ellas } \\
\text { puedan hablar de sus } \\
\text { ideas y emociones } \\
\text { acerca del momento } \\
\text { histórico que les toca } \\
\text { vivir. }\end{array}$ \\
\hline
\end{tabular}

Cuadro-resumen II

(Esquema de estrategias de enseñanza-aprendizaje y contenidos a desarrollar para potenciar la destreza productiva: "hablar" en L2 en la ESO

\begin{tabular}{|l|l|l|l|}
\hline $\begin{array}{l}\text { ¿Qué desarrollar? } \\
\text { (Subcompetencias) }\end{array}$ & $\begin{array}{l}\text { ¿Cómo desarrollar? } \\
\text { Estrategias para la } \\
\text { enseñanza/aprendizaje }\end{array}$ & $\begin{array}{l}\text { ¿Con quien } \\
\text { contar? } \\
\text { (Apoyo familia, } \\
\text { escuela, } \\
\text { comunidad, } \\
\text { etc.) }\end{array}$ & $\begin{array}{l}\text { Contexto: } \\
\text { (Área } \\
\text { trabajo: } \\
\text { Dpto., Ciclo) }\end{array}$ \\
\hline $\begin{array}{l}\text { * Puede hablar en clase, } \\
\text { que el profesor le corrija y } \\
\text { actúe como corrector. }\end{array}$ & & & \\
\hline
\end{tabular}




\begin{tabular}{|c|c|c|c|}
\hline $\begin{array}{l}\text { * Expresarse libremente } \\
\text { haciendo presentaciones de } \\
\text { varias frases referidas a sí } \\
\text { mismo o a otras personas } \\
\text { sobre aspectos tales como } \\
\text { gustos, rutina diaria, planes, } \\
\text { hechos recientes. } \\
\text { * Puede organizar sus } \\
\text { exposiciones y narrar una } \\
\text { historia corta inventada o a } \\
\text { partir de un estímulo dado: } \\
\text { libro, secuencia de } \\
\text { imágenes, película, etc. } \\
\text { * Puede, a partir de un } \\
\text { documento gráfico, como } \\
\text { una foto, describir y hablar } \\
\text { de su familia, de su pueblo } \\
\text { o ciudad o de sus } \\
\text { compañeros el día de una } \\
\text { excursión. } \\
\text { * Puede reproducir sonidos } \\
\text { y ritmos específicos de la } \\
\text { lengua extranjera a través } \\
\text { de canciones. nivel } \\
\text { * Puede representar un } \\
\text { diálogo de } \\
\text { intermedio. }\end{array}$ & $\begin{array}{l}\text { * En clase, como actividad de } \\
\text { apoyo o síntesis: } \\
\text { - Presentando un tema conocido } \\
\text { ante la clase. } \\
\text { - Exponiendo en la LE aspectos } \\
\text { de su entorno familiar y social } \\
\text { más cercano } \\
\text { - Exponiendo en la LE asuntos de } \\
\text { otras materias. } \\
\text { * En distintos ámbitos para } \\
\text { distintos públicos (clase, nivel, el } \\
\text { alumnado del centro): } \\
\text { - Cantando canciones. } \\
\text { - Recitando poemas. } \\
\text { - Representando diálogos de } \\
\text { creación } \\
\text { propia. } \\
\text { - Escenificando o haciendo teatro } \\
\text { leído. }\end{array}$ & $\begin{array}{l}\text { Familia: } \\
\text { Ayudar a los } \\
\text { estudiantes para que se } \\
\text { entrenen ante la familia } \\
\text { como presentadores de } \\
\text { exposiciones orales ante } \\
\text { el } \\
\text { público, usando una LE } \\
\\
\text { Comunidad: } \\
\text { Haciendo } \\
\text { representaciones } \\
\text { teatrales en centros } \\
\text { culturales } \\
\text { Utilizando una LE. } \\
\text { Fomentando la } \\
\text { participación a } \\
\text { través de convenios o } \\
\text { de } \\
\text { colaboraciones } \\
\text { puntuales, de EEOOII, } \\
\text { o academias de LEs } \\
\text { para que se pueda } \\
\text { facilitar la presencia en } \\
\text { el Centro de } \\
\text { profesores/as nativos. }\end{array}$ & $\begin{array}{l}\text { A realizar por el } \\
\text { Equipo Docente } \\
\text { que } \\
\text { Imparte ANLs en } \\
\text { L2. }\end{array}$ \\
\hline
\end{tabular}

Sin embargo, esta aproximación al Proyecto Atlántida no era suficiente para satisfacer nuestras demandas en relación con la necesidad de encontrar propuestas más concretas en el ámbito de las iniciativas para la mejora de la comunicación lingüística en la enseñanza bilingüe. Por ello busqué en la Red y tuve la suerte de localizar el Proyecto Educativo titulado: "Estrategias, medidas e instrumentos para la mejora de la competencia comunicativa: hacia un Plan Integrado de Lenguas del Centro", curso 2009/2010, del IES "Sánchez Lastra" de Mieres (Asturias). Por fin, esta parecía ser la horma de mi zapato. Me puse al habla con Margarita Valdés Díaz, la Coordinadora del Proyecto, a quien debo la amabilidad de facilitarme material - parte del cual se reproduce con su permiso- y unas cuantas sugerencias muy útiles. Esto era lo que llevaba largo tiempo buscando, un IES cuya Sección Bilingüe había hecho suya la propuesta del Proyecto Atlántida mediante la elaboración de un Proyecto Lingüístico de Centro propio y singular. De todos los elementos que conforman este Proyecto tan ambicioso como realista, traigo aquí a 
colación tan solo aquellos que considero imprescindibles a la hora de articular la propuesta particular que ha de ser elaborada por nuestra propia Sección Bilingüe:

a) En el ámbito de los objetivos:

Ha de quedar claro que la elaboración de un Proyecto Lingüístico de Centro debe surgir de una clara evidencia: la necesidad de la mejora de la competencia comunicativa de los alumnos/as. Este ha de ser eje sobre el que descanse todo nuestro trabajo en el aula bilingüe.

Para ello es imprescindible el trabajo coordinado de todos los docentes y estudiantes implicados en la experiencia, implicando de lleno a la Sección Bilingüe, subrayando la importancia del uso de la metodología propia del PEL (Portfolio Europeo de Lenguas) y sacando adelante el Proyecto Comenius 1.1. Un trabajo coordinado sin el cual es imposible hacer realidad un Plan de Mejora del Aprendizaje de Lenguas Extranjeras en el Centro escolar. Plan que establezca las bases para realizar un tratamiento verdaderamente integrado de las distintas lenguas del currículo; en definitiva, que incremente la mejora de la competencia comunicativa de los estudiantes de la ESO tanto en L1 como en L2.

b) En relación con la mejora del funcionamiento del Centro y de los aprendizajes de los alumnos:

Este es otro ámbito del Proyecto citado del que tomamos buena nota, con el fin de que en nuestro propio Proyecto Lingüístico de Centro se acometan las siguientes tareas:

\section{1.- La elaboración de un Plan Integrado de Lenguas:}

Esta es la primera herramienta, a partir de la cual articular todos los aspectos que incidan tanto en la organización didáctica como en el aprendizaje y uso de las lenguas en nuestro Centro.

2.- Realizar los necesarios cambios metodológicos:

Ha llegado el momento de cambiar nuestro enfoque metodológico en la enseñanza de las lenguas. Tenemos que adecuar el modelo de aprendizaje por competencias diseñado en el currículo de la ESO a partir del MCER a la enseñanza integrada de la L1 y L2.

\section{3.- Trabajar de forma bien coordinada y en la misma dirección:}

Parece obvio, pero en la práctica no lo es tanto. No resulta sencillo establecer criterios comunes en pro de la mejora de la competencia comunicativa de los alumnos que armonicen los esfuerzos que se hacen desde la Sección Bilingüe, desde el PEL o desde el Plan Lector del Centro. Por ello la expresión "trabajo coordinado" cobra en este caso todo su sentido. 
c) En relación con las medidas prácticas para incrementar la mejora de la interacción comunicativa de nuestros alumnos/as:

Somos todos los profesores/as tanto de las áreas lingüísticas como ANLs los primeros interesados en buscar nuevos enfoques metodológicos a la hora de impartir nuestras materias. Enfoques que han de centrarse en el uso de la lengua como herramienta de comunicación. Y como efecto colateral de esta mejora, nuestros estudiantes progresarán en la adquisición de otras competencias básicas como: aprender a aprender, autonomía e iniciativa personal y competencia digital entre otras.

Todos estos elementos cobran forma en la elaboración del Term Working Plan for English que el IES de referencia ha elaborado para $3^{\circ}$ de ESO en relación con First Term y que paso a reproducir toda vez que será un modelo a imitar en la confección de nuestros Planes de Trabajo correspondientes al curso 2010/2011:

\begin{tabular}{|l|l|l|l|}
\hline \multicolumn{1}{|c|}{ OBJETIVES } & UNIT & \multicolumn{1}{|c|}{ CONTENTS } & $\begin{array}{l}\text { COMMUNICATION } \\
\text { SKILLS }\end{array}$ \\
\hline $\begin{array}{l}\text { 1. Always use English for } \\
\text { communication in class. }\end{array}$ & 1 & $\begin{array}{l}\text { - Use of the Present Simple and } \\
\text { Continuous } \\
\text { - Echo questions. } \\
\text { - Infinitive of purpose. } \\
\text { - Noun formation with-er,-r, -or. } \\
\text { - Jobs and ocuppations. }\end{array}$ & $\begin{array}{l}\text { - Talk about jobs and } \\
\text { professions. } \\
\text { - Give personal information. } \\
\text { - Show interest and surprise. } \\
\text { - Keep a conversation going. } \\
\text { - Learn about opportunities to } \\
\text { improve English in summer } \\
\text { courses. }\end{array}$ \\
\hline $\begin{array}{l}\text { 2. Get used to the routine } \\
\text { of } \\
\text { using Portfolio to } \\
\text { organize } \\
\text { daily work. }\end{array}$ & 2 & $\begin{array}{l}\text { - Past simple. Regular and irregular } \\
\text { verbs. }\end{array}$ & $\begin{array}{l}\text { - Talk about past situation. } \\
\text { - Describe past journeys. }\end{array}$ \\
\hline $\begin{array}{l}\text { - Use of time connectors. } \\
\text { 3. Encourage situations } \\
\text { and task ( both oral an } \\
\text { written) } \\
\text { that allow students to } \\
\text { actively } \\
\text { practice a variety of } \\
\text { vocabulary, structures and } \\
\text { funtions. }\end{array}$ & 3 & $\begin{array}{l}\text { - Time adverbials. } \\
\text { - Means of transport. }\end{array}$ & $\begin{array}{l}\text { - Different ways of expressing } \\
\text { future time. }\end{array}$ \\
\hline $\begin{array}{l}\text { 4. Reflect about our own } \\
\text { learning. }\end{array}$ & & $\begin{array}{l}\text { - Introduction to relative clauses. } \\
\text { - Most common expressions to } \\
\text { make } \\
\text { invitations and replay to them. } \\
\text { - Environment vocabulary }\end{array}$ & $\begin{array}{l}\text { - Make plans for future. } \\
\text { - Invite someone and answer to } \\
\text { someone's invitation. } \\
\text {-Collect and organise ideas for } \\
\text { writing. }\end{array}$ \\
\hline $\begin{array}{l}\text { 5.- Learn about other } \\
\text { schools } \\
\text { systems and different } \\
\text { sociocultural aspects of } \\
\text { English espeaking } \\
\text { countries. }\end{array}$ & 4 & $\begin{array}{l}\text { - Use of the Present Perfect with } \\
\text { for and since. }\end{array}$ & $\begin{array}{l}\text { - Describe one's exprerienices. } \\
\text { - Compare poeple and objects. } \\
\text { - Express one's opinions }\end{array}$ \\
\hline
\end{tabular}




\begin{tabular}{|l|l|l|}
\hline CLIL & $\begin{array}{l}\text { and } \\
\text { Art classes }\end{array}$ & $\begin{array}{l}\text { oral and written- to explore } \\
\text { basic } \\
\text { structures and vocabulary } \\
\text { related } \\
\text { to specific topic. } \\
\text { - Use expressions learnt in the } \\
\text { English class to acomplish tasks } \\
\text { such as class presetantions, oral } \\
\text { and written reports, etc }\end{array}$ \\
\hline
\end{tabular}

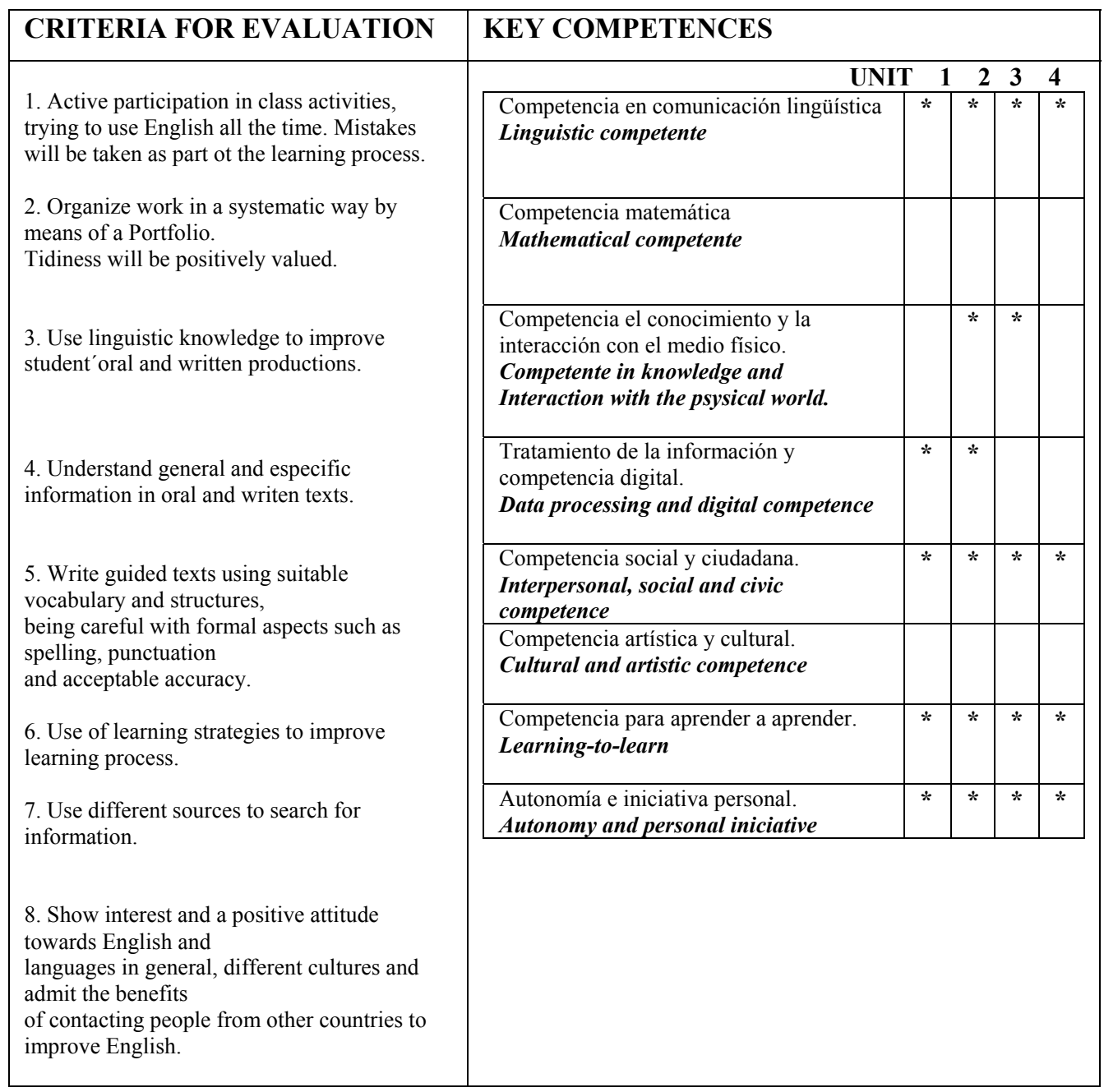




\section{ELABORACIÓN DE PROPUESTAS PROPIAS VÁLIDAS PARA MEJORAR LA COMPETENCIA LINGÜÍSTICA DE LOS ALUMNOS/AS EN L2}

Teniendo presentes los modelos consultados, ahora corresponde al autor elaborar aquellas propuestas didácticas que entiende han de resultar útiles para el profesorado de las Secciones Bilingües que se esfuerza en mejorar los índices de interacción comunicativa de los estudiantes de la ESO. Se trata de llegar al último nivel de concreción, de descender a la práctica docente y de apostar una metodología plenamente renovada. A tal fin se ofrecen cinco modelos de actividades, cada una de las cuales van aumentando de forma progresiva en dificultad, y que en su conjunto son un instrumento útil para el fin que se persigue.

a) Inicio: Learning languages discussing questions.

Como introducción al proceso, me parece oportuno que los alumnos/as contesten las siguientes cuestiones:

1.- Do you think that opportunities to speak are more important than being corrected?

Why do you say that?

2.- What do you think the consequences of having other lessons at school taught in English would be?

3.- What are the advantages and disadvantages of having all your errors corrected?

4.- What is the best way to learn a language, in your opinion?

5.- How much does how you should learn a language vary from person to person.

6.- Do you believe that parents in your country put too much emphasis on learning English? Why do you have that opinion?

7.- Do you think your government is doing enough to promote foreigh languages?

8.- How has the importance of English in your country changed over the past 50 years?

9. What can be done to improve English language education for kids, do you think?

10.- Do you think that the teaching of foreign languages is getting better or worse?

What makes you say that?

11.- In your opinion, how important is starting a language young?

12.- When is the best age for learning a foreign language efficiently?

13.- What is the best way to help a baby learn its own language fast and well?

14.- Why do matters talk to children who are too young to understand? 
15.- Why do people talk to babies in a particular way? Do you think that is true all round the world?

16.- What did you think about analysing and breaking down the language in the way you just did with the Greek word roots? Is it interesting? Will il help you to learn? Do you think that is the same for everybody?

b) Debates and discussion:

En un segundo paso proponemos un esquema para práctica de los debates en L2 en clase. Se trata de un modelo que puede ser modificado y adaptado por cada profesor a la realidad de su aula:

1. - General instructions and topics for group discusion or debate:

A debate is a kind of contest where you must support your argument and refute your opponent's argument with logical reasoning and rebuttals by giving facts and evidence. It is best to choose topics of interest to the participants. In order to support your argument, you may have to make statements which are against your own opinion. It is best never to agree with the opposite side until after the debate.

Topics:

1.- Money invested in space travel should be better invested in helping the poor and needy.

2.- Experimenting on animals in laboratories is cruel.

3.- Genetic engineering is nothing but a violation of the laws of nature.

4.- Cloning could have disasterou disastrous consequences for mankind in the future.

5.- The energy crisis is "necessary" to maintain the world balance power.

6.- Man is own worst enemy.

7.- There is always a price to pay in protecting the environment.

8.- In the $21^{\text {st }}$ century mankind will regress to primitive levels of existence.

9.- Animals should not be kept in captivity.

10- It is right to meddle with Nature. 


\section{2.- Grouping and Procedure:}

All the participants will be divided into 2 groups, A and B each group must decide which side of the argument to take: FOR or AGAINST the proposed topic of discussion.

FOR and AGAINST side will be seated opposite one another. It may be a good idea to prepare your side of the argument first: delegate a person in each group to take note of ideas (in summarized form) put forward by the group. Carry out a 'brainstorming session' beforehand if necessary. By consultating ideas, your side of the argument will flow more easily. All relevant ideas and opinions can then be mentioned one by one during the debate. The chairperson will start the debate by summarizing the situation at heart. This followed by:

1.- a 3-minute constructive speech from each side

2.- two o three 2 -minute rebuttals from each side turn by turn

3.- a 3-minute concluding speech from each side

4.- questions from the floor - the audience can address questions to both sides after de debate.

c) English as a $2^{\text {nd }}$ language:

Se trata de una modalidad de debate con el que se pretende que los alumnos/as interactúen y se expresen con la mayor corrección posible en la L2. Aportamos un ejemplo al respecto:

1.- Topic: Men and Women. Equal at last?

2.- Aim: Improve conversational skills when supporting a view. equal.

3.- Activity: Debate about the question of whether men and women are truly

4.- Level: Upper-intermediate to advanced.

5.- Outline:

- Review language used when expressing opinions, disagreeing, making comments on other person's point of view, etc.

- Write a few ideas on the board to encourage discussion of the equality between men and women: the workplace, the home, government, etc.

- Ask students' responses, divide class up into two groups. One group arguing that equality has been achieved for women and on that feels that women have not yet attained true equality to men. Idea: Put students into the group with the opposite opinion of what they seemed to believe in the warm-up conversation.

- Give students worksheets including ideas pro and con. Have students develop arguments using ideas on the worksheets as a springboard for further ideas and discussion.

- Once students have prepared their opening arguments, begin with the debate. Each team has 5 minutes to present their principal ideas.

- Have students prepare notes and make rebuttal to the expressed opinions. 
- While the debate is in progress, take notes on common errors made by the students.

- At the end of the debate, take time for a short focus on common mistakes. This is important, as students should not be too involved emotionally and therefore will be quite capable of recognizing language problems.

\section{6.- Giving reasons and offering explanations:}

\section{1.- Yes, women are now equal to men:}

- Many governments have both male and female representatives.

- Many companies are now owned or managed by women.

- A lot of progress has been made since the 1960 s.

- Television series now portray women as successful career makers.

- Men now share in the raising children and household responsibilities.

- Many important laws have been passed to ensure equality in the workplace.

- In many places, a married coupled can choose whether the man or the woman takes leave from work to look after the newly arrived baby,

- People aren't discussing equality anymore. It has become a reality.

- Have ever heard of Margaret Thatcher?

\section{2.- Excuse me? Women still have a long way to go before they are equal} to men:

- Women still earn less than men in may work situations.

- Women are still portrayed in a superficial manner in many televison television's shows.

- Look at the international sporting. How many professional professionals' female leagues are as successful as their male counterparts?

- Most governments still are made up in their majority of men.

- We are having this debate because women are not equal. Otherwise, there would be no need to discuss the matter.

- Women are often not given enough responsibility based on the possibility that they might become pregnant.

- The number of sexual harassment suits have increase over the past ten years

- Hundreds of years of history can't have been changed in a mere 30 odd years.

- Have you ever watched Bay Watch?

d) General topics: (to encourage cooperation in groups):

Mediante este tipo de actividades se trata de fomentar la interacción comunicativa de cada alumno en L2 a través del trabajo cooperativo en grupo. Por medio de sus intervenciones en relación con un tópico general se pretende reforzar sus destrezas lingüísticas como usuario de la LE. Vemos lo 
aquí expuesto mediante ejemplos que pueden ser localizados en la Red y que potencian el uso de las NNTT de la Información y de la Comunicación en el aprendizaje de las lenguas extranjeras:

\section{General Topic: Roman Britain}

2. Level: Bilingual Teaching 1s ESO

\section{3.-Introduction:}

In the $1^{\text {st }}$ century B. C. ,Rome was no longer a city. It was the capital of a great empire. Romans ruled land from France to North Africa. Look at this map (pulsa el ratón aquí, en esta webtask: http://lolaceituno.googlepages.com/romanbritain) and name some current (actuales) countries which were under the influence of Rome.

4.- General questions:

What do you know about Britain before the Romans invide invaded it? Click here to find the answer to these questions:

a) Who ruled Britain before the Romans invide invaded it?

b) What year did the Romans invade Britain and which Emperor sent de Army?

c) Why did the Romans build forts?

d) Why did Emperor Hadrian build a wall? What is the name of it? How long did the Romans take in building it? Click here and you will find more information.

e) Can we find remains of the Roman roads in Britain today?

f) Name the three types of enemies the Romans had to fight against in Britain.

g) Where did rich people live? Describe their houses.

h) Where can you find these houses in Great Britain now?

i) How do you know that Manchester was a Roman town?

j) Name three activities that Romans enjoyed.

k) Where in Britain can you find the most famous Roman baths?

m) Tell us the number of years that Romans stayed in Britain. If you look at this timeline (Click here) you can calculate.

\section{1.- General topic: Ancient Egypt}

2.- Level: Bilingual Teaching $2^{\text {nd }}$ ESO

3.- Introduction:

It is a webtask (http://lolaceituno.googlepages.com/ancientegypt) we are going to have a look at some of the main characteristics of Ancient Egyptian civilization: setting, monuments, burials, goods and goddesses, paintings, writing. You'll fill attracted to it!

4.- General questions:

a) Click here and look at the map of Egypt. Click on the different places and answer these questions:

1- Egypt is to the west of which sea?

2.- What is the "old" name of the capital of Egypt? What can you fine close to the capital? 
3.- What is called "black land". What is the different between black land and red land?

4.- What can you fin to the west of Nile River?

b) The Egyptians use a kind of writing calls hieroglyphics. Let's see what we know about this writing:

1.- Where did Egyptians love writing?

2.- What was discovered in 1799 and why was it so important for Archaelogists?

3.- Who could translate the hieroglyphics in the end an how?

4.- Click here to write your name in hieroghyphics-

c) What about the writing and paintings of ancient Egyptians:

1.- Look at the real examples of wall paintings and explain what topics Egyptians reflected on their paintings.

2.- If you are interested in mummification, click here and watch the process of embalming and wrapping a body.

3.-Click on Ramesses's beard and do the quiz. When you finish, complete the worksheet. Good luck!!!

e) Classroom activities that engage the main: strategies for Empowering Students.

Se trata de presentar a los alumnos/as una serie de actividades para el fomento de sus destrezas comunicativas en L2. Son un modelo de propuesta didáctica desarrollada por la University of Illinois Extensión y que el docente puede localizar en la web: http://urabanext.illinois.edu/ce. Son iniciativas graduadas: primary, intermediate, and upper level. Para nuestra ejemplificación tomamos una de las propuestas para aplicar en $4^{\circ}$ de ESO:

1.- Topic: What's in a Name?

2.- Purpose:

* To help students reflect on their self-worth and enhance their selfconcepts.

* To promote the students' appreciation of their individuality, ethnicity, and cultural heritage.

* To foster the integration of the language arts skills.

3.- Focus:

* Development and use of new and/or unfamiliar vocabulary words.

* Differential use of synonyms, antonyms, and homonyms.

4.- Integration of Content/Subject Areas:

* Language Arts

* Career Development

* Character Building

* English

5.- Strategy/Activity: 
Students will use their metacognitive skills, knowledge, vocabulary, and creative thinking and writing skills to complete the following activity. This activity can be done individually or in cooperative teams of two or four.

Each student will write his or her name vertically down the left-hand side of the sheet of paper, writing each letter separately. The student will think of a word or phrase to match each letter. The words or phrase must correspond with each letter and be arranged to formulate a positive statement or prediction about the person:

\section{Example:}

$\begin{array}{lll}\text { LIN } & \text { JOSE } & \text { MAE } \\ \text { Lovable } & \text { Jazzy } & \text { Making } \\ \text { Involved } & \text { Obstetrician } & \text { An } \\ \text { Neigbour } & \text { Sailing } & \text { Effort } \\ & \text { Everywhere } & \end{array}$

Students are to develop a paragraph or story based on their name descrptions. These are to be shared orally with the class. Students are to explain the process they used in developing the descriptors for their individual names. They must also justify why or how the descriptors accurately represent what or who they are or plan to become. This activity may take more than one class period to complete. This activity is an excellent homeworknemen assignment for bilingual students and their parents who are learning English as a second language.

\section{6.- Extended Research:}

Ask the students to read the statements from Alice's Adventures in Wonderland: "With a name like yours, you might any shape, almost":

- Ask the students to determinate the two characters having the dialogue, where (the setting), why this statement was made, and the meaning.

- Have students research other work by Lewis Carroll and compare the style of writing he used in Alice's Adventures in Wonderland to them.

- Ask students to read and share other books in which the characters have unusual or significant names. Have them tell how these names reflect the character traits and/or values of these characters.

7.- $\underline{\text { Assessment: }}$

- How effectively did the students process and apply the directions?

- How was the student's use of metacognitive skills exhibited?

- In what ways did students demonstrate knowledge of their ethnic or cultural heritage?

- How did students express pride and satisfaction with themselves as individuals? 
- How dis students demonstrate their skill and knowledge base in writing, thinking, listening, and speaking? bank?

- In what ways did this activity enhance the students' vocabulary

\section{CONCLUSIONES}

No cabe duda de que pueden existir muchas formas de afrontar las carencias en interacción comunicativa en L2 que en la actualidad tienen nuestros alumnos/as de las Secciones Bilingües. A lo largo de estas páginas hemos ofrecido a los docentes implicados en estas tareas una de ellas. Cada cual conoce de primera mano la realidad de su Centro y las necesidades de sus estudiantes, por ello no existen recetas ad hoc. Lo importante es que sea cual sea el modelo elegido, las tareas que se realicen con los alumnos de la ESO se centren en el fomento de sus destrezas comunicativas tanto en la L1 como en la L2.

Todo el éxito de nuestra empresa dependerá de que los docentes seamos capaces de ofertar a nuestros estudiantes la oportunidad de utilizar las lenguas en múltiples situaciones de comunicación con un claro objetivo social: que reconozcan la disparidad de sus niveles de competencia lingüística y se apliquen a superar sus carencias. Es decir, que al final del curso, las diversas evaluaciones realizadas arrojen un saldo positivo en relación con los objetivos previstos. La nuestra no es tarea fácil, pero no por ello hemos de desanimarnos. Lograr que los alumnos/as aumenten sus niveles de comprensión lectora en los textos leídos en L2, que mejoren su expresión oral y escrita, que amplíen su vocabulario o que interactúen oralmente de forma fluida es todo un reto. El itinerario está trazado, ahora solo queda que de cara al curso 2014/2015 los recursos humanos y materiales para recorrerlo sean holgados para llegar a la meta sin síntomas de cansancio.

\section{REFERENCIAS BIBLIOGRÁFICAS}

ÁLVAREZ, A y DEL RÍO, P. (1990). Actividad y lenguaje: el diseño cultural como marco para la enseñanza del inglés como Lengua Extranjera. Comunicación, lenguaje y educación 7-8

CEJA, Plan de Fomento del Plurilingüísmo en Andalucía. BOJA, $\mathrm{n}^{\circ}$ 65, de 5.4.2005

CUMMINS, J y CORSON, D. (1997). Bilingual Education. International Encyclopedia of Language and Education. Vol. 5.

GENNESSE, F. (1987).earning through two languages:studies of Immersion and Bilingual education. Rowly, M. A. Newbury House.

KRASHEN, S. P. (1985). The input Hypothesis: issues and implications. New York: Longman. 
MADARIAGA, J. (2010). Aportaciones de la investigación psicolingüística a experiencias de comunicación educativa en varias lenguas. Revista Electron, 12. www.sc.ehu.es/pewpanes/relectron/n12

MOYA OTERO, T y LUENGO ORCAJO, M. (2009). Proyecto Atlántida. MEOPAEE.

PÉREZ CALVO, M. (2010). Los beneficios que conlleva impartir disciplinas no lingüísticas (ANLs) en Francés (Lengua Extranjera) en Secciones Bilingües. Universidad de Zaragoza. Dpto. de Didáctica de C. H. y Sociales. Internet: 5.6.2010.

SERRA, J. M. (1989). Resultados académicos y desarrollo cognitivo en un programa de inmersión dirigido a escolares de nivel sociocultural bajo. Infancia $y$ aprendizaje 47.

VILA, I. (2010). "Qué puede aportar la educación bilingüe a la educación lingüística del Siglo XXI." Avances en supervisión educativa 13 